\title{
Niflumic acid disrupts marine spermatozoan chemotaxis without impairing the spatiotemporal detection of chemoattractant gradients
}

\author{
Adán Guerrero ${ }^{1,2}$, Jesús Espinal ${ }^{3}$, Christopher D. Wood ${ }^{1}$, Juan M. Rendón ${ }^{4}$, Jorge Carneiro ${ }^{2}$, \\ Gustavo Martínez-Mekler ${ }^{3,5}$ and Alberto Darszon ${ }^{1, *}$
}

${ }^{1}$ Departamento de Genética del Desarrollo y Fisiología Molecular, Instituto de Biotecnología, Universidad Nacional Autónoma de México, Cuernavaca, Morelos, 62210, México

${ }^{2}$ Instituto Gulbenkian de Ciência, Rua da Quinta Grande, 6 2780-156, Oeiras, Portugal

${ }^{3}$ Instituto de Ciencias Físicas, Universidad Nacional Autónoma de México, Cuernavaca, Morelos, 62210, México

${ }^{4}$ Facultad de Ciencias, Universidad Autónoma del Estado de Morelos, Cuernavaca, Morelos, 62209, México

${ }^{5}$ Dipartimento di Fisica e Astronomia, Università degli Studi di Firenze, Via Sansone 1-I 50019, Firenze, Italia

*Author for correspondence (darszon@ibt.unam.mx)

Accepted 30 December 2012

Journal of Cell Science 126, 1477-1487

(c) 2013. Published by The Company of Biologists Ltd

doi: $10.1242 / j c s .121442$

\section{Summary}

In many broadcast-spawning marine organisms, oocytes release chemicals that guide conspecific spermatozoa towards them through chemotaxis. In the sea urchin Lytechinus pictus, the chemoattractant peptide speract triggers a train of fluctuations of intracellular $\mathrm{Ca}^{2+}$ concentration in the sperm flagella. Each transient $\mathrm{Ca}^{2+}$ elevation leads to a momentary increase in flagellar bending asymmetry, known as a chemotactic turn. Furthermore, chemotaxis requires a precise spatiotemporal coordination between the $\mathrm{Ca}^{2+}$-dependent turns and the form of chemoattractant gradient. Spermatozoa that perform $\mathrm{Ca}^{2+}$-dependent turns while swimming down the chemoattractant gradient, and conversely suppress turning events while swimming up the gradient, successfully approach the center of the gradient. Previous experiments in Strongylocentrotus purpuratus sea urchin spermatozoa showed that niflumic acid (NFA), an inhibitor of several ion channels, drastically altered the speract-induced $\mathrm{Ca}^{2+}$ fluctuations and swimming patterns. In this study, mathematical modeling of the speract-dependent $\mathrm{Ca}^{2+}$ signaling pathway suggests that NFA, by potentially affecting hyperpolarization-activated and cyclic nucleotidegated channels, $\mathrm{Ca}^{2+}$-regulated $\mathrm{Cl}^{-}$channels and/or $\mathrm{Ca}^{2+}$-regulated $\mathrm{K}^{+}$channels, may alter the temporal organization of $\mathrm{Ca}^{2+}$ fluctuations, and therefore disrupt chemotaxis. We used a novel automated method for analyzing sperm behavior and we identified that NFA does indeed disrupt chemotactic responses of L. pictus spermatozoa, although the temporal coordination between the $\mathrm{Ca}^{2+}$ dependent turns and the form of chemoattractant gradient is unaltered. Instead, NFA disrupts sperm chemotaxis by altering the arc length traveled during each chemotactic turning event. This alteration in the chemotactic turn trajectory disorientates spermatozoa at the termination of the turning event. We conclude that NFA disrupts chemotaxis without affecting how the spermatozoa decode environmental cues.

Key words: $\mathrm{Ca}^{2+}$ signaling, Chemotaxis, Niflumic acid, Sperm

\section{Introduction}

In many species, including mammals, spermatozoa are guided towards the oocyte by gradients of chemoattractants released from the female gamete or, more commonly, their investments (Kaupp et al., 2008; Darszon et al., 2011). This chemotactic behavior is widely documented in marine invertebrates that undergo external fertilization (Miller, 1985; Ward et al., 1985). Their spermatozoa redirect themselves by a signaling pathway that translates local changes in the chemoattractant concentration into a periodic modulation of the path curvature (Friedrich and Jülicher, 2007). The redirection is driven by $\mathrm{Ca}^{2+}$-dependent increases in flagellar bending asymmetry during the turns, and decreases in asymmetry during the straighter swimming episodes (Miller and Brokaw, 1970; Kaupp et al., 2003; Wood et al., 2005; Shiba et al., 2008). Notably, the path curvature does not strictly follow the $\left[\mathrm{Ca}^{2+}\right] \mathrm{i}$, as spermatozoa remain swimming on straighter paths at elevated $\left[\mathrm{Ca}^{2+}\right]_{i}$ (Wood et al., 2005; Shiba et al., 2008; Böhmer et al., 2005; Guerrero et al., 2010a). Recent findings indicate that the sperm swimming pathway is controlled by the $\left[\mathrm{Ca}^{2+}\right]$ i time derivative (Alvarez et al., 2012), however the responsible molecular mechanisms involved are not fully understood.

The decapeptide speract (GFDLNGGGVG) is one of the most widely studied members of the sperm-activating peptide family that modulate sea urchin sperm motility (Suzuki, 1995) (reviewed in Darszon et al., 2008). This peptide, purified from Strongylocentrotus purpuratus eggs, also cross-reacts with spermatozoa from Lytechinus pictus sea urchins (Hansbrough and Garbers, 1981; Suzuki et al., 1981; Suzuki and Garbers, 1984). Current models propose that the binding of speract to its receptor promotes the synthesis of cGMP that activate $\mathrm{K}^{+}$ selective and cyclic nucleotide-gated channels (KCNG) leading to membrane potential $\left(V_{\mathrm{m}}\right)$ hyperpolarization (Galindo et al., 2005; Strünker et al., 2006; Bönigk et al., 2009). This $V_{\mathrm{m}}$ change 
first induces a $\mathrm{pH}_{\mathrm{i}}$ increase (Nishigaki et al., 2001; Nishigaki et al., 2004), stimulates hyperpolarization-activated and cyclic nucleotide-gated channels (HCN) (Gauss et al., 1998), removes the inactivation of voltage-gated $\mathrm{Ca}^{2+}$ channels $(\mathrm{CaV})$ (Strünker et al., 2006; Granados-Gonzalez et al., 2005), and facilitates $\mathrm{Ca}^{2+}$ extrusion by $\mathrm{Na}^{+} / \mathrm{Ca}^{2+}$ exchangers (NCKX) (Jayantha Gunaratne and Vacquier, 2007; Su and Vacquier, 2002; Nishigaki et al., 2004). The opening of $\mathrm{HCN}$ and the influx of $\mathrm{Na}^{+}$contribute to $V_{\mathrm{m}}$ depolarization, and concomitant increases in $\left[\mathrm{Ca}^{2+}\right]_{\mathrm{i}}$ and $\left[\mathrm{Na}^{+}\right]_{\mathrm{i}}$ further depolarize $V_{\mathrm{m}}$. This fast transient increase in flagellar $\left[\mathrm{Ca}^{2+}\right]_{\mathrm{i}}$ has been associated with the transient increases in flagellar bending that prompt sea urchin spermatozoa to undergo a turning event. It has been proposed that the $\left[\mathrm{Ca}^{2+}\right]_{\mathrm{i}}$ increases could lead to the opening of $\mathrm{Ca}^{2+}$-regulated $\mathrm{Cl}^{-}$ channels $(\mathrm{CaCC})$ and/or $\mathrm{Ca}^{2+}$-regulated $\mathrm{K}^{+}$channels $(\mathrm{CaKC})$, which would then contribute to hyperpolarize the $V_{\mathrm{m}}$ again, removing inactivation from $\mathrm{CaV}$ channels and opening $\mathrm{HCN}$ channels (Wood et al., 2007; Espinal et al., 2011). It is thought that this series of events is then cyclically repeated generating a sequence of $V_{\mathrm{m}}$-dependent turns.

The absence of external $\mathrm{Ca}^{2+}$ or the presence of certain $\mathrm{Ca}^{2+}$ permeable cation channel inhibitors disrupts sperm chemotaxis (Kaupp et al., 2003; Wood et al., 2005; Guerrero et al., 2010a; Wood et al., 2007; Yoshida et al., 2002). Recently, we discovered that L. pictus spermatozoa undergo chemotaxis in response to an experimentally generated speract gradient (Guerrero et al., 2010a). Interestingly, even though a similar speract gradient stimulates $S$. purpuratus spermatozoa to redirect their swimming paths with the stereotypical sequence of turns interspersed with periods of straighter swimming, it does not induce chemotaxis. Thus, the $\mathrm{Ca}^{2+}$-dependent turning episodes and the interspersed periods of straighter swimming are necessary, but not sufficient, for chemotaxis.

Sperm chemotaxis of marine invertebrates requires the strict coupling of the $\mathrm{Ca}^{2+}$ oscillations with the direction or polarity of the chemoattractant gradient (Böhmer et al., 2005; Shiba et al., 2008; Guerrero et al., 2010a; Kashikar et al., 2012). We found that L. pictus spermatozoa are able to suppress the onset of $\mathrm{Ca}^{2+}$ fluctuations while swimming toward the center of a speract gradient. However, after crossing the positive-to-negative speract gradient inversion point, the $\mathrm{Ca}^{2+}$-dependent turning events occur. Therefore, a fundamental element of sea-urchin sperm chemotaxis lies in the ability to suppress $\mathrm{Ca}^{2+}$-mediated increases in flagellar curvature while swimming in ascending (positive) chemoattractant gradients.

Niflumic acid (NFA) is a nonsteroidal anti-inflammatory drug that blocks or modifies the gating of many ion channels including HCN, CaCC and CaKC (Pacaud et al., 1989; White and Aylwin, 1990; Janssen and Sims, 1992; Akbarali and Giles, 1993; Espinosa et al., 1998; Greenwood and Large, 1995; Hogg et al., 1994; Satoh and Yamada, 2001; Li et al., 2008; Cheng and Sanguinetti, 2009). Treatment of $S$. purpuratus spermatozoa with NFA increases the duration, amplitude and interval between successive speract-triggered $\left[\mathrm{Ca}^{2+}\right]_{\mathrm{i}}$ fluctuations (Wood et al., 2007; Wood et al., 2003). These alterations in $\left[\mathrm{Ca}^{2+}\right]_{\mathrm{i}}$ dynamics have strong repercussions on $S$. purpuratus sperm motility; in the presence of NFA, the speract-induced turns are more pronounced and of greater duration (Wood et al., 2007).

In a companion article (J.E., A.D., A.G. and G.M.-M., unpublished) we investigated how NFA could modify the $\mathrm{Ca}^{2+}$ fluctuation dynamics using a logical network model for the speract-activated $\mathrm{Ca}^{2+}$ signaling pathway developed by Espinal et al. (Espinal et al., 2011). Here, based on the results of this model under conditions in which it can reproduce the experimental findings of Wood et al. (Wood et al., 2003; Wood et al., 2007), we anticipate that in the presence of speract gradients, $\left[\mathrm{Ca}^{2+}\right]$ i fluctuations would no longer be biased towards the descending gradients in the presence of NFA. To investigate this hypothesis, we exposed L. pictus spermatozoa to NFA to modify the speract-induced ion permeability changes and study their impact on chemotaxis.

\section{Results}

Blockage of NFA-sensitive channels in a model of the speract signaling pathway alters the temporal organization of $\left[\mathrm{Ca}^{2+}\right]_{i}$ fluctuations

Inhibition of $\mathrm{HCN}, \mathrm{CaKC}$ and $\mathrm{CaCC}$ in the model leads to changes in $\left[\mathrm{Ca}^{2+}\right]_{\mathrm{i}}$ oscillations, which are characterized by larger amplitudes, a temporal shift of the peaks and troughs and higher average concentration levels, when compared to the speractstimulated oscillations in untreated conditions (Fig. 1A) (J.E., A.D., A.G. and G.M.-M., unpublished). To try to understand how the predicted alteration in $\mathrm{Ca}^{2+}$ oscillations induced by NFA would impinge on swimming behavior and chemotaxis, we mapped the time series simulations of $\mathrm{Ca}^{2+}$ (Fig. 1A), produced by the model under the experimental conditions of Wood et al. onto a circle representing the trajectory of a spermatozoon swimming in the plane (see Fig. 1B for details) (Wood et al., 2007). Taking into account a dominant four period component in the oscillations in Fig. 1A (J.E., A.D., A.G. and G.M.-M., unpublished), we mapped time into a circular path ensuring that the spatial period corresponds to a temporal period of 4 time units. Furthermore, in order to envisage speract gradient effects, we shifted time so that a resemblance is attained between the time course of mean $\mathrm{Ca}^{2+}$ values for the untreated speract-stimulated sperm (Fig. 1C) and the spatiotemporal positioning of chemotactic turns along the circular path observed in L. pictus spermatozoa (Guerrero et al., 2010a). We set the appearance of $\mathrm{Ca}^{2+}$ peaks in the untreated speractstimulated sperm in the Fig. 1C, left panel, so that they were biased towards descending phases of the speract gradient $(\varphi: 0-\pi)$. Notice the similarity of this figure with the experimental $\left[\mathrm{Ca}^{2+}\right]_{\mathrm{i}}$ fluctuations in Fig. 4C, which shows chemotactic turns experienced by the spermatozoa of L. pictus after exposure to a speract gradient (supplementary material Fig. S4C).

When the time series representing the $\mathrm{Ca}^{2+}$ dynamics of NFAtreated speract-stimulated sperm were mapped in the same way, the bias in the timing of the peaks in $\mathrm{Ca}^{2+}$ oscillations towards the descending gradients was lost (Fig. 1C, right panel). Thus, according to our findings (Guerrero et al., 2010a) the logical model predicts that NFA treatment would disrupt chemotaxis in L. pictus spermatozoa by relocating the $\mathrm{Ca}^{2+}$ peaks that control chemotactic turns further around the circular path relative to the chemoattractant gradient.

\section{NFA disrupts sperm chemotaxis}

To investigate the hypothesis that NFA affects chemotactic responses by altering the temporal organization of $\left[\mathrm{Ca}^{2+}\right] \mathrm{i}$ oscillations, and thus relocating chemotactic turns, we analyzed the swimming paths of NFA-treated L. pictus spermatozoa in a speract gradient. Fig. 2 illustrates sperm motility and sperm responses to a speract gradient in the absence (Speract) and presence of $30 \mu \mathrm{M}$ of NFA (Speract+NFA). A speract gradient with a Gaussian concentration distribution was generated via the 
photolysis of $10 \mathrm{nM}$ caged speract with a $200 \mathrm{~ms}$ UV flash through an optical fiber (Guerrero et al., 2010a), during which only $5-10 \%$ is photo-released after UV irradiation (Tatsu et al., 2002). Before UV irradiation, spermatozoa swam in circles with near-constant radii (Speract: $23.8 \pm 0.9 \mu \mathrm{m}$; Speract+NFA: $26.7 \pm 1.5 \mu \mathrm{m} ; \quad P=0.27, n>45$, Wilcoxon test; Fig. 2A,C). Immediately after UV irradiation they generated a stereotypical motility response of turns interspersed with the straighter swimming episodes in both experimental conditions: Speract and Speract+NFA (Fig. 2B,C; supplementary material Fig. S2 left panels). Previous studies showed that, under identical experimental conditions, the chemotactic response of L. pictus spermatozoa is restricted to the first $3 \mathrm{~s}$ following speract uncaging, which is spatially delimited to the region $40-170 \mu \mathrm{m}$ from the center of the speract gradient (Guerrero et al., 2010a). Within these spatial and temporal limits the speract gradient remains essentially unchanged after the UV pulse. A Gaussian distribution was fitted to the radial profile of the UV light scattered at the water-glass interface and used to estimate the shape of the speract gradient created by uncaging. The temporal evolution of the speract gradient was then computed based on the
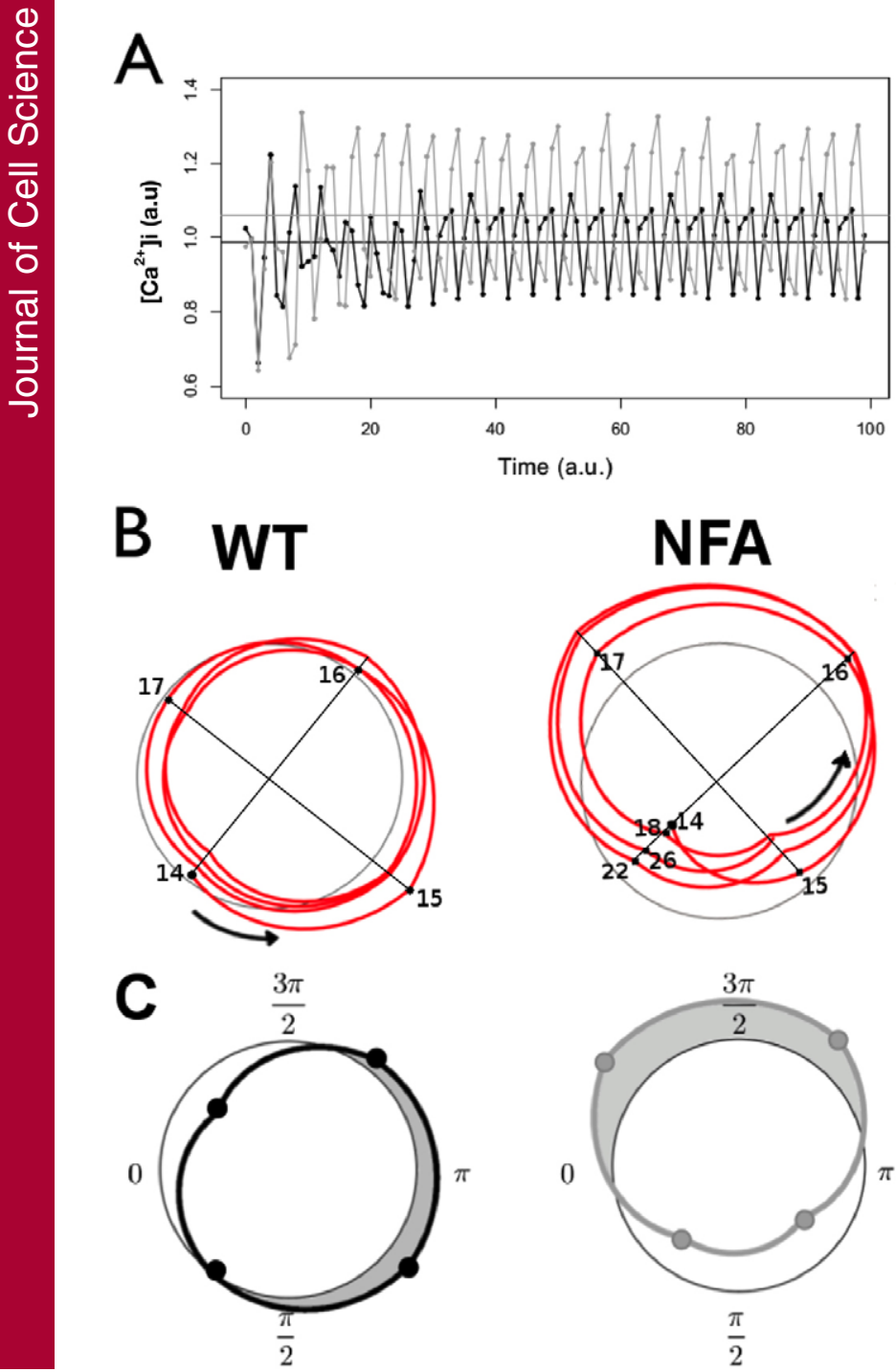

\section{B WT}
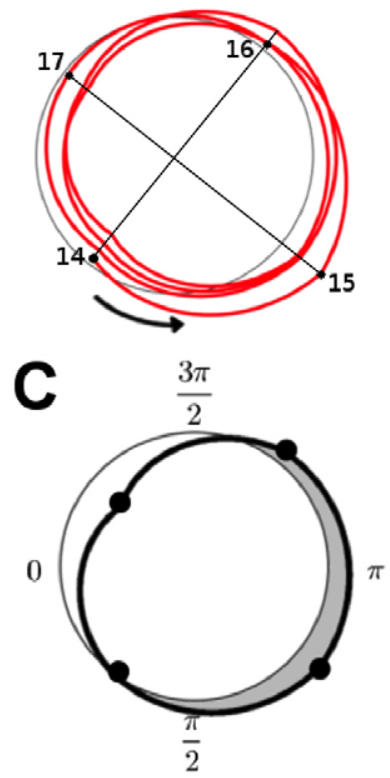

NFA
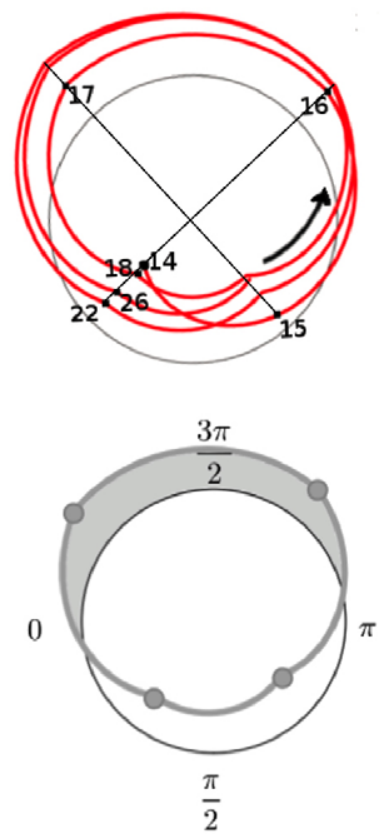

diffusion coefficient of the peptide (supplementary material Fig. $\mathrm{S} 3$; note that at $100 \mu \mathrm{m}$ from the center of the chemoattractant gradient the speract concentration was constant during the initial 3 s period post-UV stimulation).

We assayed chemotaxis in the L. pictus spermatozoa that were swimming at $40-150 \mu \mathrm{m}$ from the center of the speract gradient from three seconds before (as control) and throughout three seconds after UV irradiation (Fig. 3). In both experimental conditions, Speract and Speract+NFA, unstimulated spermatozoa showed a distribution of linear equation chemotaxis index (LECI) values centered around zero $\left(\mathrm{LECI}_{\text {mean }}=1.1 \pm 0.2 \mu \mathrm{m} / \mathrm{s}\right.$ and $0.7 \pm 0.6 \mu \mathrm{m} / \mathrm{s}$, respectively) (Yoshida et al., 2002; Guerrero et al., 2010a). When exposed to the speract gradient alone the cell distribution shifted towards the positive range indicating a biased sperm re-localization towards the gradient center: $\mathrm{LECI}_{\text {mean }}=13.5 \pm 2.5 \mu \mathrm{m} / \mathrm{s} \quad$ (Fig. 3; supplementary material Movie 1). In contrast, NFA-treated spermatozoa respond to the speract gradient with half of the cells experiencing negative chemotaxis: (Fig. 3; supplementary material Movie 2). A shift of the left tail of the distribution of LECI values towards the negative range was observed: bimodal distribution $(P=0.03$, Hartigans' Dip test for unimodality). The overall mean was found close to zero: $-3.0 \pm 2.9 \mu \mathrm{m} / \mathrm{s}$; Fig. 3. Statistically insignificant differences were found when comparing the overall distribution of NFAtreated speract-stimulated spermatozoa against unstimulated spermatozoa ( $P=0.35$, Wilcoxon test). We conclude that NFA disrupts the capability of $L$. pictus spermatozoa to bias their motility behavior towards the center of the speract gradient.

Fig. 1. Simultaneous inhibition of $\mathrm{HCN}, \mathrm{CaKC}$ and $\mathrm{CaCC}$ by NFA in a logical model of speract signaling pathway alters the temporal organization of $\left[\mathrm{Ca}^{2+}\right]_{i}$ oscillations and potentially disrupts the chemotactic bias of $\mathbf{C a}^{2+}$-dependent turns. (A) Time dependence of the average value, taken over 1000 independent random initial conditions, of $\left[\mathrm{Ca}^{2+}\right]_{\mathrm{i}}$ determined from our network dynamics as a response to speract. Black: untreated (wild-type) spermatozoa; grey: NFA-treated spermatozoa. The dark-grey horizontal line indicates the time average of the above over 100 wild-type generated points; the light-grey horizontal line is the equivalent time average of the NFA case. The effect of NFA was modeled by setting the logical variables representing $\mathrm{HCN}, \mathrm{CaKC}$ and $\mathrm{CaCC}$ to zero.

(B) Intersections of the straight lines with the red curve segments (some of which are shown as black dots) are the polar coordinate plots of the $\left[\mathrm{Ca}^{2+}\right]_{\mathrm{i}}$ data shown in A from time step 14 to 26; wild type on the left and NFAtreated on the right. The radius, measured from the straight line intersections, encodes the values of $\left[\mathrm{Ca}^{2+}\right]_{i}$ as in $\mathrm{A}$, with a scale set by the grey circle fixed to the corresponding gray horizontal values in A. The time-related angular coordinate $\varphi_{F}$ has units such that a time step in A corresponds to $\pi / 2$, hence the spatial period of the circumference is spanned in four time-steps. Red circular segments give an indication of the point succession ordered in the direction determined by the arrows; numbers indicate the time step value of the labeled point according to A. (C) Points are time-averaged values of the wild-type (left panel) and NFA-treated (right panel), $\left[\mathrm{Ca}^{2+}\right]_{\mathrm{i}}$ series shown in A, taken at four time-step intervals. As an example, in B, 4 of the 25 points that intervene in the time-average are labeled explicitly. Shaded regions are representative of the distribution of points for short time scales. The initial time in the polar coordinate representation is chosen in the left panel so that the likelihood of having an averaged $\left[\mathrm{Ca}^{2+}\right]_{\mathrm{i}}$ wild-type peak is higher for values of $\varphi_{F}$ between 0 and $\pi$, i.e. in the descending phase of the chemoattractant gradient. In the presence of NFA (right panel) under the same setup, the peak bias of the $\left[\mathrm{Ca}^{2+}\right]_{\mathrm{i}}$ fluctuations determined by the model appears shifted to the interval in between $\pi$ and $2 \pi$ (right panel), i.e. to an ascending speract gradient. 


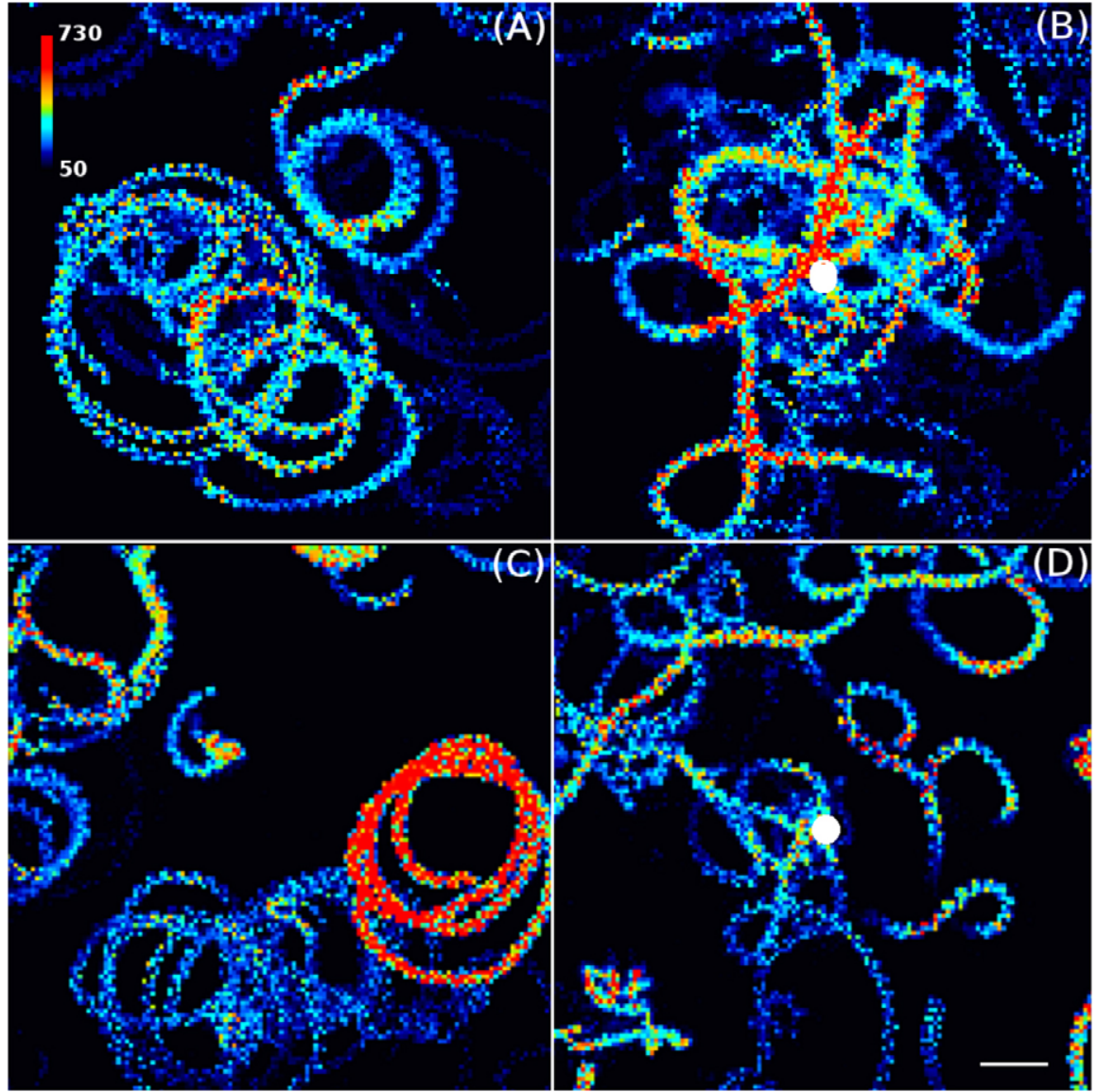

Fig. 2. Typical speract-induced motility changes. Time projections showing sperm trajectories and fluo-4 fluorescence $3 \mathrm{~s}$ before $(\mathbf{A}, \mathbf{C})$ and $3 \mathrm{~s}$ after (B,D) the $200 \mathrm{~ms}$ UV irradiation of $10 \mathrm{nM}$ caged speract $(\mathrm{CS})$ in ASW $(\mathrm{A}, \mathrm{B})$ or in ASW containing, $30 \mu \mathrm{M}$ NFA (C,D). The white dots show the UVirradiated area that corresponds to the center of the speract gradient. A pseudo-color scale represents maximum (red) and minimum (blue) relative fluo-4 fluorescence. Scale bar: $25 \mu \mathrm{m}$. Examples of singlecell trajectories are shown in supplementary material Fig. S2.
NFA does not affect how sperm detect the speract gradient We previously reported that L. pictus spermatozoa experience chemotactic responses to the speract concentration gradient due the selective triggering of $\mathrm{Ca}^{2+}$ dependent turns while swimming down the speract gradient (Guerrero et al., 2010a). Contrastingly, when spermatozoa from the sea urchin $S$. purpuratus were exposed to the same experimental conditions they failed to undergo chemotaxis due the spatially unbiased triggering of $\mathrm{Ca}^{2+}$ dependent turning events relative to the speract gradient (Guerrero et al., 2010a). Hence, the ability to couple the $\left[\mathrm{Ca}^{2+}\right]_{\mathrm{i}}$ oscillations with the direction of the chemoattractant concentration gradient is fundamental for sperm chemotaxis. We therefore investigated whether NFA disrupts chemotaxis in $L$. pictus sperm through disruption of their mechanism for selectively suppressing chemotactic turns in ascending chemoattractant gradients.

We analyzed the behavior of sperm during the period between the UV-stimulated uncaging event and the first $\left[\mathrm{Ca}^{2+}\right]_{\mathrm{i}}$ fluctuation. In these conditions, the duration of exposure to an ascending speract gradient in each spermatozoon will depend on its position at the end of the period of UV irradiation. Initially, increases in speract concentration created during the $200 \mathrm{~ms}$ of UV exposure will be sensed by all spermatozoa in the field as an ascending gradient of speract. At the end of the $200 \mathrm{~ms}$ of UV irradiation spermatozoa will find themselves in either a newlyformed positive or negative gradient. The spermatozoa that immediately experience a descending speract gradient after the uncaging event will sense lower rates of speract binding than those that encounter an ascending speract gradient. If the mechanism to suppress the $\left[\mathrm{Ca}^{2+}\right]_{\mathrm{i}}$ increase in ascending chemoattractant gradients is robust, then the two sperm states (ascending gradient after UV, or ASC, and descending gradient after UV, or DESC) will differ in the delay they experience before undergoing the first $\left[\mathrm{Ca}^{2+}\right]_{\mathrm{i}}$ fluctuation.

Our working hypothesis is that DESC spermatozoa will experience at the end of the uncaging event a fall in the rate of speract binding, cGMP synthesis and $\mathrm{K}^{+}$-dependent $V_{\mathrm{m}}$ hyperpolarization; consequently initiating the signaling cascade that leads to the $\left[\mathrm{Ca}^{2+}\right]_{\mathrm{i}}$ increase and the flagellar motility changes. However, ASC spermatozoa will initially swim within a continuing ascending speract gradient, experiencing a relative delay before reaching the gradient inversion point at which the chemotactic turn-promoting signaling mechanism is activated. Thus ASC sperm will show significantly increased intervals between the end of the UV exposure and the initiation of the first $\left[\mathrm{Ca}^{2+}\right]_{\mathrm{i}}$ fluctuation than DESC sperm (Guerrero et al., 2010a).

We determined the direction of individual spermatozoa at the end of the UV irradiation period ( $\left.\varphi_{\text {UVend }}\right)$, and correlated this value with the time required for the onset of the first $\left[\mathrm{Ca}^{2+}\right]_{\mathrm{i}}$ fluctuation $\left(\tau_{F 0}\right.$; Fig. 4A,B,D,E). Values of $\varphi_{\text {UVend }}$ between 0 and $\pi$ identify DESC spermatozoa, and $\varphi_{\text {UVend }}$ values between $\pi$ and $2 \pi$ identify ASC spermatozoa. Untreated speract-stimulated ASC spermatozoa significantly increased the average $\tau_{F 0}$ compared to untreated DESC spermatozoa (410 \pm 43 versus $162 \pm 10 \mathrm{~ms}$; Fig. 4A,B). This relatively extended delay to the first $\left[\mathrm{Ca}^{2+}\right]_{i}$ fluctuation in ASC spermatozoa compared to DESC spermatozoa 
Chemotactic Index (LECI)

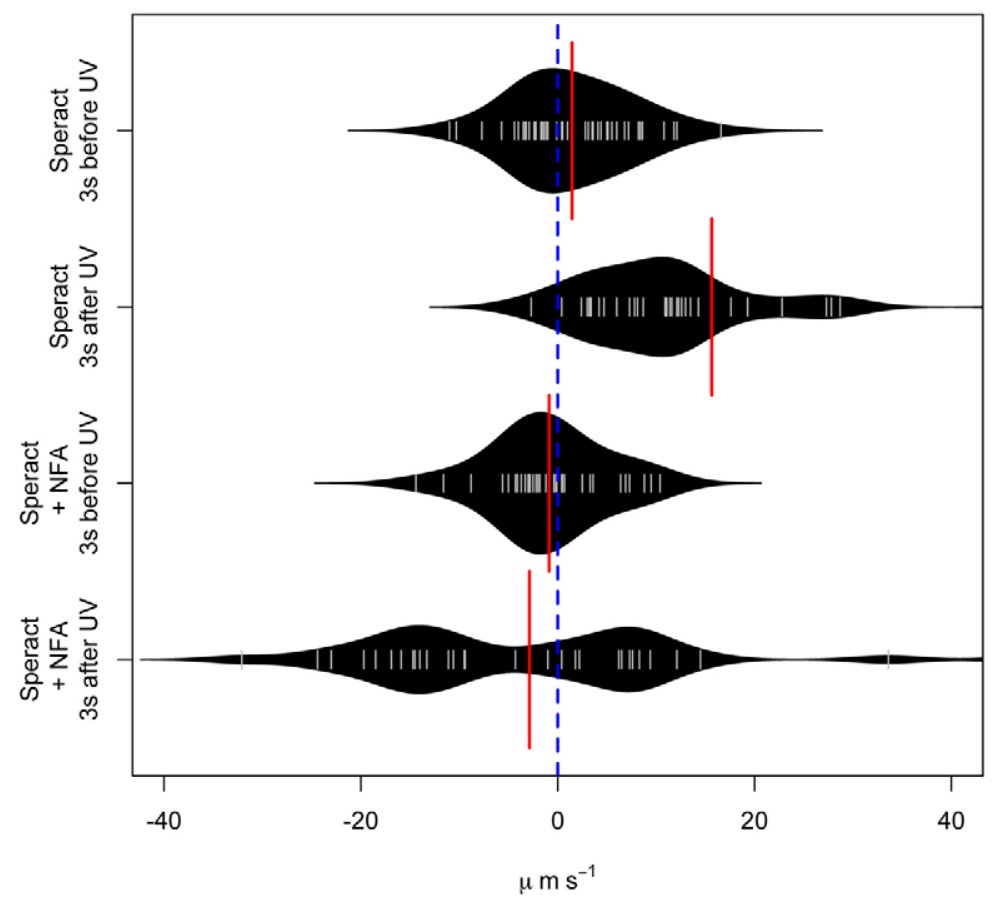

Fig. 3. NFA disrupts sperm chemotaxis. Distributions of LECI values calculated from $3 \mathrm{~s}$ before to $3 \mathrm{~s}$ after exposure to a speract gradient alone (Speract), or in speract and NFA (Speract+NFA), $n>20$. Red solid lines indicate the mean values and the dashed blue line shows the zero-crossing values. was also seen in NFA-treated spermatozoa (410 \pm 50 versus 200 \pm 50 ms, respectively; Fig. 4D,E).

The increased delay seen in the ASC sperm population is sufficient to retard the onset of the $\left[\mathrm{Ca}^{2+}\right]_{i}$ elevation until the sperm enters into the descending phase of the speract gradient. To determine if NFA modifies the relative sperm positioning on the speract gradient we measured the position of individual spermatozoa relative to the direction of the speract gradient at the beginning of the first $\left[\mathrm{Ca}^{2+}\right]_{\mathrm{i}}$ fluctuation $\left(\varphi_{F 1}\right)$. In both experimental conditions, Speract and Speract+NFA, spermatozoa undergo the first $\left[\mathrm{Ca}^{2+}\right]_{\mathrm{i}}$ fluctuation near to the minimum of the speract gradient (the farthest point from the center; Fig. $4 \mathrm{C}, \mathrm{F}$; Table 1).

The distribution of the positions of individual spermatozoa at the beginning of subsequent $\left[\mathrm{Ca}^{2+}\right]_{\mathrm{i}}$ fluctuations $\left(\varphi_{F}\right)$ was skewed towards the minimum of the speract gradient in both experimental conditions, Speract and Speract+NFA (Fig. 4C,F; Table 1). These data show that NFA neither alters the signaling mechanism that suppress the increase in $\left[\mathrm{Ca}^{2+}\right]_{\mathrm{i}}$ as spermatozoa are swimming in a positive chemoattractant gradient, nor alters the position at which the $\left[\mathrm{Ca}^{2+}\right]_{\mathrm{i}}$ fluctuations occur relative to the center of the chemoattractant gradient.

The relationship between the timing of the $\left[\mathrm{Ca}^{2+}\right]_{\mathrm{i}}$ fluctuations and the shape of the chemical gradient determines the positioning of the turn-and-run motility response (Böhmer et al., 2005; Shiba et al., 2008; Guerrero et al., 2010a; Guerrero et al., 2010b; Kashikar et al., 2012). Supplementary material Fig. S4 shows the correlation of the direction of individual spermatozoa at the end of the UV irradiation period with the time required for the onset of the first turning event $\left(\tau_{T 0}\right)$. As expected, ASC spermatozoa showed significantly increased intervals between the end of the UV exposure and the initiation of the first turning event than DESC spermatozoa $(310 \pm 43$ versus $60 \pm 43 \mathrm{~ms}$, supplementary material Fig. S4A,B). Similar behavior was shown for those spermatozoa treated with NFA (410 \pm 43 versus $160 \pm 43 \mathrm{~ms}$, supplementary material Fig. S4D,E). Curiously, without NFA the average $\tau_{T 0}$ was significantly lower than the average $\tau_{F 0}$ (compare Fig. 4B; supplementary material Fig. S4B). This discrepancy may be due to our algorithm detecting the onset of turns prior to the point at which flagellar $\left[\mathrm{Ca}^{2+}\right]_{\mathrm{i}}$ levels rise above the threshold of detection, given that basal levels of flagellar $\left[\mathrm{Ca}^{2+}\right]_{\mathrm{i}}$ are undetectable in swimming L. pictus sea urchin spermatozoa (Guerrero et al., 2010a).

We next examined if the distribution of the positions of spermatozoa at the onset of the first turning event had a biased preference, and found that it was skewed towards the minimum of the speract gradient in both experimental conditions, Speract and Speract+NFA (supplementary material Fig. S4C; Table 2).

In summary, we find no evidence that NFA interferes with signaling mechanisms that suppress the triggering of an increase in the $\left[\mathrm{Ca}^{2+}\right]_{\mathrm{i}}$ while spermatozoa are swimming up a speract gradient, nor that it alters the position of the $\mathrm{Ca}^{2+}$-dependent turning events relative to center of the chemoattractant gradient.

\section{Niflumic acid treatment increases the arc length traveled during each turning event but not its duration}

The effect of the chemotactic turn is to reorient the spermatozoon along a new trajectory that directs it towards the source of a chemoattractant via a transient episode of relatively straightened swimming trajectory. We next investigated whether the disruption of chemotaxis by NFA is due to a defect in the alignment of this straighter swimming path relative to the gradient center after a chemotactic turn. Path misalignment can, in principle, follow a turn performed with an abnormal angular velocity, an abnormal time period and/or along an abnormal path curvature. In the presence of NFA the turns were more pronounced (Fig. 2D; supplementary material Fig. S2B; Fig. 5A) due to an increased arc length traveled during each 
A)

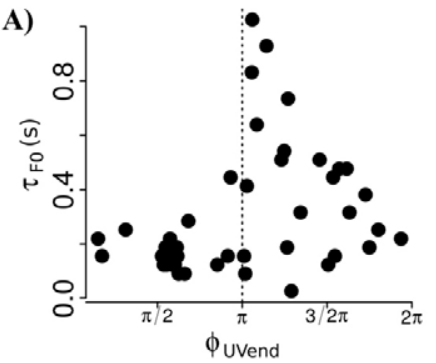

B)
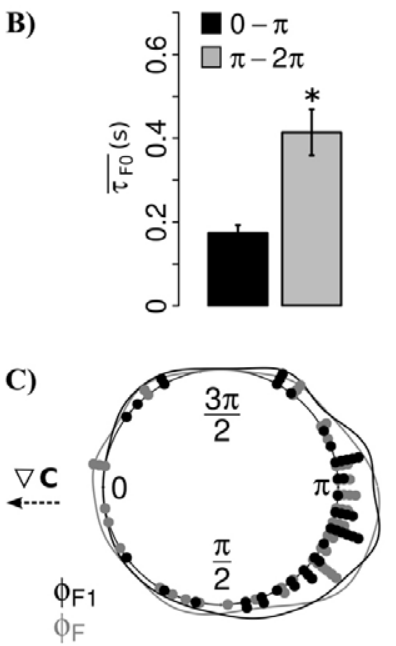

D)

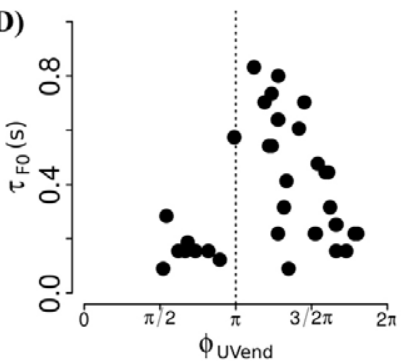

E)

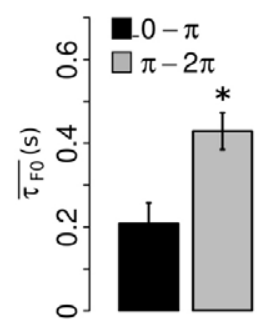

F)

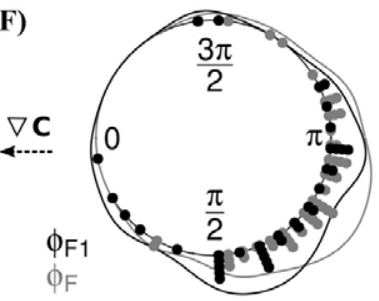

Fig. 4. NFA does not affect the mechanism that delays the onset of the $\left[\mathrm{Ca}^{2+}\right]_{i}$ fluctuations until reaching a descending phase of the speract gradient. Spermatozoa exposed to the speract gradient alone (Speract; A-C) or in speract and NFA (Speract+NFA; D-F). (A,D) Relative sperm position on the speract gradient at the end of the UV irradiation period ( $\left.\varphi_{\text {UVend }}\right)$ versus the delay to the onset of the first $\left[\mathrm{Ca}^{2+}\right]_{\mathrm{i}}$ fluctuation $\left(\tau_{F 0}\right)$. (B,E) Average delay of the onset of the first $\left[\mathrm{Ca}^{2+}\right]_{\mathrm{i}}$ fluctuation $\left(\tau_{F 0}\right)$ of ASC (gray) or DESC (black) spermatozoa $(* P<0.001$, Wilcoxon-test, $n \geq 9)$. (C,F) Circular distribution of the relative sperm positioning on the speract gradient at the onset of the first $\left[\mathrm{Ca}^{2+}\right]_{\mathrm{i}}$ fluctuation $\left(\varphi_{F 1}\right)$ or at the onset of the subsequent fluctuations $\left(\varphi_{F}\right) ; n \geq 36$.

turning event $(\mathbf{U})$ from $0.6 \pm 0.05$ to $0.85 \pm 0.11 \%$ of revolution per turn (Fig. 5B), although in both conditions the turns were of the same duration $\left(\tau_{T} \approx 0.5 \mathrm{~s}\right.$; Fig. $\left.5 \mathrm{C}\right)$. The increase in $\mathbf{U}$ due to NFA treatment was correlated with a significant increase in the angular velocity during each turning event (from $\mathrm{w}_{T}=12.7 \pm 0.4$ to $14.0 \pm 0.5 \mathrm{rad} / \mathrm{s}$; Fig. 5D). Interestingly NFA does not alter the average interval between $\left[\mathrm{Ca}^{2+}\right]_{\mathrm{i}}$ fluctuations (Speract:

Table 1. Rayleigh test of circular distribution ( $P$-values) - the $\left[\mathrm{Ca}^{2+}\right]_{i}$ fluctuations are biased towards the range $[\pi / 2, \pi]$

\begin{tabular}{llllll}
\hline & \multicolumn{5}{c}{ Specific mean direction } \\
\cline { 2 - 6 } & - & 0 & $\pi / 2$ & $\pi$ & $3 \pi / 2$ \\
\hline Speract, $\phi_{F 1}$ & 0 & 0 & 0.93 & 1 & 0.07 \\
Speract, $\phi_{F}$ & 0 & 0 & 0.98 & 1 & 0.02 \\
Speract+NFA, $\phi_{F 1}$ & 0 & 0.0005 & 1 & 1 & 0.047 \\
Speract+NFA, $\phi_{F}$ & 0 & 0 & 1 & 1 & 0.0005
\end{tabular}

The alternative hypothesis $\mathrm{H}_{0}$ is a unimodal distribution with unknown $\phi$ mean (second column: '-'), otherwise $\mathrm{H}_{0}$ is a unimodal distribution with a specified $\phi$ mean direction (columns 3 to 6 : ' 0 ', ' $\pi / 2$ ', ' $\pi$ ', ' $3 \pi / 2$ ').

Table 2. Rayleigh test of circular distribution ( $P$-values) - the $\mathrm{Ca}^{2+}$-dependent turning events are biased towards the range $[\pi / 2, \pi]$

\begin{tabular}{llllll}
\hline & \multicolumn{5}{c}{ Specific mean direction } \\
\cline { 2 - 6 } & - & \multicolumn{1}{c}{0} & $\pi / 2$ & \multicolumn{1}{c}{$\pi$} & $3 \pi / 2$ \\
\hline Speract, $\phi_{T 1}$ & 0.02 & 0.3 & 1 & 0.7 & 0.04 \\
Speract, $\phi_{T}$ & 0 & 0.0004 & 1 & 1 & 0.001 \\
Speract+NFA, $\phi_{T 1}$ & 0 & 0.002 & 1 & 1 & 0.0001 \\
Speract+NFA, $\phi_{T}$ & 0 & 0 & 1 & 1 & 0.0001
\end{tabular}

The alternative hypothesis $\mathrm{H}_{0}$ is a unimodal distribution with unknown $\phi$ mean (second column: '-'), otherwise $\mathrm{H}_{0}$ is a unimodal distribution with a specified $\phi$ mean direction (columns 3 to 6 : ' 0 ', ' $\pi / 2$ ', ' $\pi$ ', ' $3 \pi / 2$ ').

$0.75 \pm 0.05 \mathrm{~s}, \quad$ Speract+NFA: $0.78 \pm 0.04 \mathrm{~s}$ ), nor the average interval between turns (Speract: $0.89 \pm 0.05 \mathrm{~s}$, Speract+NFA: $0.90 \pm 0.07 \mathrm{~s}$; supplementary material Fig. S5). In addition, the distribution of the straighter swimming episodes of the NFAtreated spermatozoa was shifted towards negative values and centered close zero (Fig. 5D). Furthermore, the duration of the straighter swimming episodes was the same in both experimental conditions $\left(\tau_{S} \approx 0.5 \mathrm{~s}\right.$; Fig. $\left.5 \mathrm{~F}\right)$.

\section{NFA-treated spermatozoa are disoriented at the beginning of the straight-swimming episodes}

The increased arc length traveled during each turning event displayed by the NFA-treated spermatozoa (Fig. 5) suggests that they are disoriented at the onset of the period of straighter swimming. We determined the relative positioning of individual spermatozoa with respect to the speract gradient at the onset of the first straight swimming episode $\left(\varphi_{S 1}\right)$, or at the onset of the subsequent straight swimming episodes $\left(\varphi_{S}\right)$. Chemotactic spermatozoa experience the onset of the straighter swimming episodes in the ascending phase of the speract gradient (Fig. 6, left; Table 3). On the contrary, NFA-treated spermatozoa displayed a phase shift of $\pi / 2$ at the onset of the straighter swimming episodes (close to the nearest point to the center of the speract gradient; Fig. 5, right; Table 3). This incorrect positioning drives NFAtreated spermatozoa away from the center of the chemoattractant gradient, disrupting the chemotactic response in these cells.

\section{Discussion}

The binding of speract to its receptor initially triggers $V_{\mathrm{m}}$ hyperpolarization by opening cGMP-gated $\mathrm{K}^{+}$channels (KCNGs) which leads to a $\mathrm{pH}_{\mathrm{i}}$ alkalinization and then to $\left[\mathrm{Ca}^{2+}\right]_{\mathrm{i}}$ increases (Darszon et al., 2011). It has been proposed that spermatozoa swimming up a positive chemoattractant gradient will experience increasing rates of chemoattractant binding and receptor activation, leading to sustained membrane hyperpolarization due to the opening of KCNG channels. This hyperpolarized state is sustained until spermatozoa enter negative chemoattractant gradients, at which point the membrane depolarizes leading to the opening of $\mathrm{CaV}$ channels (Guerrero et al., 2010a; Darszon et al., 2008; Bönigk et al., 2009; Strünker et al., 2006; Guerrero et al., 2010b; Galindo et al., 2007; Cook et al., 1994). We found that NFA does not apparently perturb this putative chemoattractant gradient decoding mechanism, indicating that the molecular targets of NFA are downstream of the components regulating the initial $\mathrm{Ca}^{2+}$ burst.

Our current model for speract-induced signaling events proposes that the initial KCNG channel-mediated hyperpolarization of the 


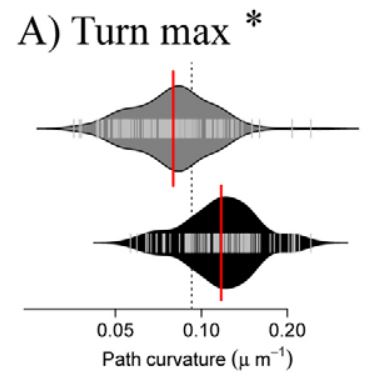

B) Revolutions per turn $(\%)^{*}$

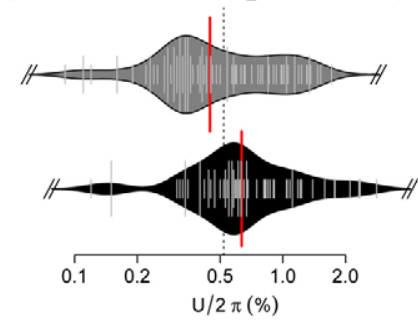

C) Turn duration
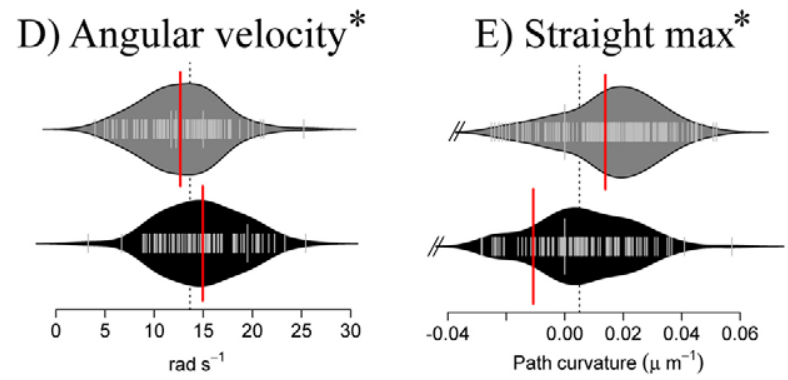

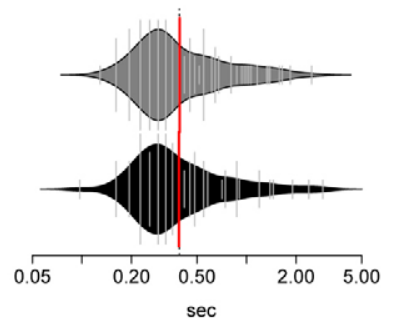

Speract $\square$ Speract + NFA

F) Straight duration

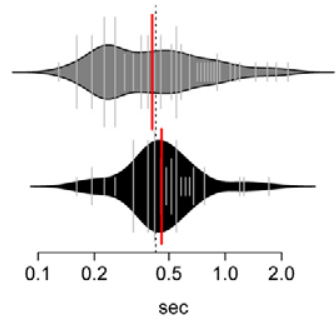

Speract $\square$ Speract + NFA
Fig. 5. NFA increases the arc length traveled during each turning event but not its duration. $(\mathbf{A}, \mathbf{B})$ Distribution of the maximum curvature values (A, $n \geq 107$ ), arc length traveled (B; as a percentage of revolutions per turn: $\mathrm{U} / 2 \pi, n \geq 41$ ) during the speract-induced turning event. (C) Duration of the turning events ( $n \geq 75$ ). (D) Distribution of the mean angular velocity of the turning events $(n \geq 75)$. (E) Distribution of the minimum curvature values: 'the most straighter swimming episodes' ( $n \geq 110)$. (F) Duration of the straighter swimming episodes $(n \geq 47)$. ${ }^{*} P<0.01$, Wilcoxon test. Speract: $10 \mathrm{nM}$ CS; Speract+NFA: $10 \mathrm{nM}$ $\mathrm{CS}+30 \mu \mathrm{M}$ NFA. Red solid lines indicate the mean. Note the log scale of A-C,E. sperm membrane potential releases $\mathrm{CaV}$ channels from inactivation, and stimulates the activity of HCN channels (Darszon et al., 2008). The opening of $\mathrm{HCN}$ channels repolarizes $V_{\mathrm{m}}$, leading to opening of $\mathrm{CaV}$ channels. The KCNG channel-mediated hyperpolarization could theoretically reach the $\mathrm{K}^{+}$equilibrium potential $\left(E_{\mathrm{K}+} \approx-95 \mathrm{mV}\right.$ ), depending on the degree of stimulation. In Arbacia puctulata spermatozoa the hyperpolarization following exposure to chemoattractant gradually increased over five orders of magnitude of chemoattractant concentration, showing a maximal response at $25 \mathrm{nM}$ (Strünker et al., 2006). Since in our experimental assay we employed $10 \mathrm{nM}$ of caged speract, and the photo-uncaging efficiency is in the range of 5-10\% (Tatsu et al., 2002), the higher speract concentration used to stimulate the spermatozoa of L. pictus should be in the picomolar range (Guerrero et al., 2010a). This means that the initial KCNG-mediated hyperpolarization will be within the resting $V_{\mathrm{m}}=-40 \mathrm{mV}$ and $-95 \mathrm{mV}$, and needs to be

\section{Relative sperm position on the speract gradient}
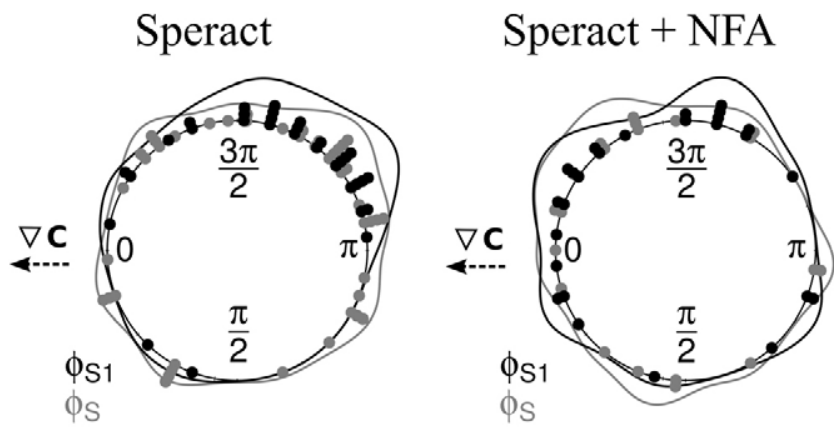

Fig. 6. NFA-treated spermatozoa display a phase shift of $\pi / 2$ to the onset of straighter swimming episodes. Circular distribution of the relative positioning of spermatozoa with respect to the speract gradient at the onset of the first turning episodes $\left(\varphi_{S 1}\right)$ or at the onset of the subsequent episodes $\left(\varphi_{S}\right)$. Spermatozoa were exposed to the speract gradient alone (left) or in the presence of speract and NFA (right). enough to remove $\mathrm{CaV}$ channel inactivation. In this physiological $V_{\mathrm{m}}$ range only a fraction of the HCN will open (Gauss et al., 1998) to promote $\mathrm{Na}^{+}$influx (the $I_{\mathrm{h}}$ current) and generate the initial repolarization required to open $\mathrm{CaV}$ channels (however, once opened, $\mathrm{Ca}^{2+}$ influx will further contribute to the depolarization). In rod photoreceptor cells NFA promotes a shift of the $V_{\mathrm{m}}$ dependence of HCN towards more negative values (Satoh and Yamada, 2001). It is possible that in NFA-treated spermatozoa the $I_{\mathrm{h}}$ current will contribute less to the $V_{\mathrm{m}}$ re-depolarization phase of the speract signaling pathway (Fig. 7A,i). If this is true, other depolarizing elements will be required to compensate the diminished redepolarizing role of $I_{\mathrm{h}}$ in NFA-treated spermatozoa (otherwise the initial $\left[\mathrm{Ca}^{2+}\right]_{\mathrm{i}}$ transient should never occur after NFA treatment). Since ZD7288, an HCN blocker, only delays the $\left[\mathrm{Ca}^{2+}\right]_{\mathrm{i}}$ transient triggered by speract (Nishigaki et al., 2004), it seems feasible that other currently uncharacterized depolarizing elements, such as CatSper, participate in this phase of re-depolarization.

As the resting potential of sea urchin sperm is $\approx-40 \mathrm{mV}$ (Strünker et al., 2006; González-Martínez and Darszon, 1987; Schackmann et al., 1981), and the $\mathrm{Cl}^{-}$equilibrium potential $E_{\mathrm{Cl}^{-}}$ is $-69.5 \pm 7.5 \mathrm{mV}$ (Wood et al., 2007), the opening of $\mathrm{Cl}^{-}$ channels would result in an influx of $\mathrm{Cl}^{-}$and a hyperpolarization

Table 3. Rayleigh test of circular distribution ( $P$-values) NFA-treated spermatozoa display a phase shift of $\pi / 2$ to the onset of the straighter swimming episodes

\begin{tabular}{llllll}
\hline & \multicolumn{5}{c}{ Specific mean direction } \\
\cline { 2 - 6 } & \multicolumn{1}{c}{-} & 0 & $\pi / 2$ & $\pi$ & $3 \pi / 2$ \\
\hline Speract, $\phi_{S 1}$ & 0 & 0.02 & 0 & 0.98 & 1 \\
Speract, $\phi_{S}$ & 0.007 & 0.03 & 0.006 & 0.97 & 0.99 \\
Speract+NFA, $\phi_{S 1}$ & 0.01 & 0.93 & 0.005 & 0.07 & 0.99 \\
Speract+NFA, $\phi_{S}$ & 0.08 & 0.97 & 0.12 & 0.03 & 0.88
\end{tabular}

The alternative hypothesis $\mathrm{H}_{0}$ is a unimodal distribution with unknown $\phi$ mean (second column: '-'), otherwise $\mathrm{H}_{0}$ is a unimodal distribution with a specified $\phi$ mean direction (columns 3 to 6 : ' 0 ', ' $\pi / 2$ ', ' $\pi$ ', ' $3 \pi / 2$ '). 

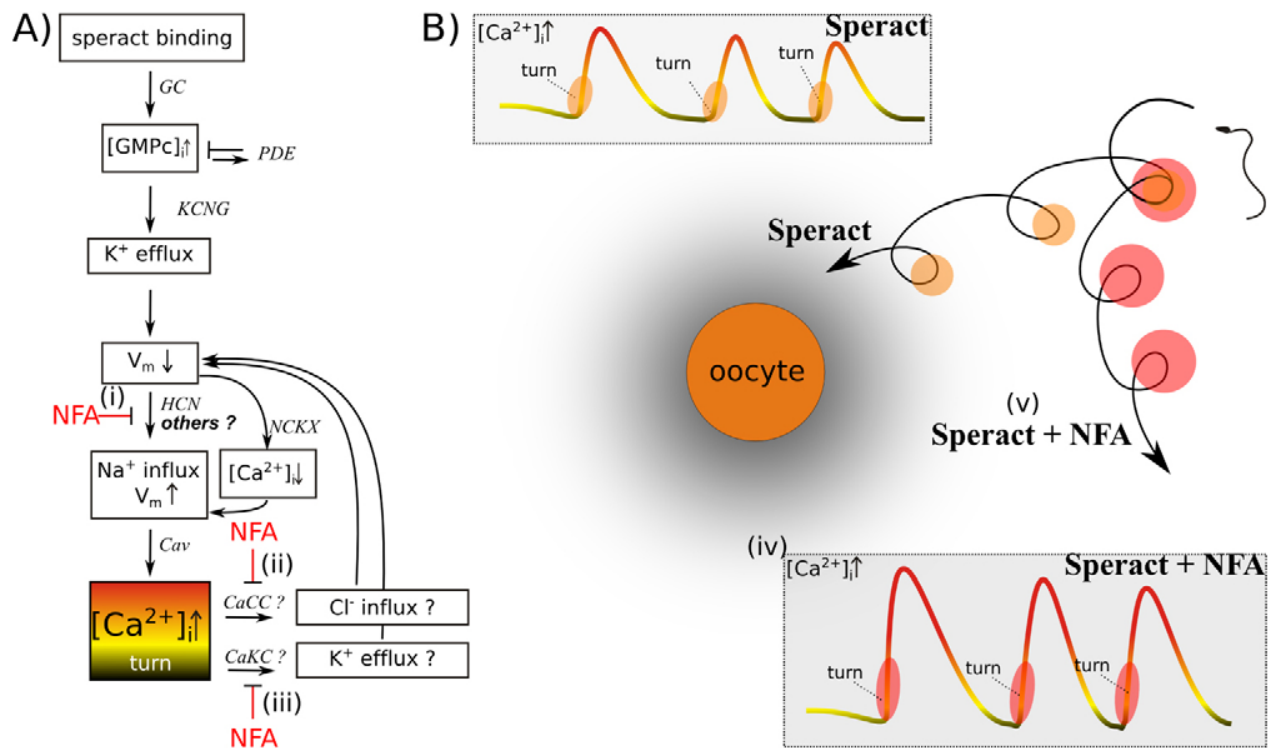

Fig. 7. Schematic representation of how perturbations to speract signaling disrupt chemotaxis. (A) Possible NFA targets and their putative role in the speract signaling pathway. In the range 1-100 $\mu \mathrm{M}$ : NFA reduces the $I_{\mathrm{h}}$ current of HCN channels (i) (Satoh and Yamada, 2001; Cheng and Sanguinetti, 2009). Interestingly neither NFA, nor the HCN blocker ZD7288 prevents the increase in the $\left[\mathrm{Ca}^{2+}\right]_{\mathrm{i}}$ triggered by speract (Nishigaki et al., 2004), suggesting that other molecular elements could contribute to the $V_{\mathrm{m}}$ re-polarization required for $\mathrm{CaV}$ channel opening. NFA blocks CaCC (ii) (Pacaud et al., 1989; White and Aylwin, 1990; Janssen and Sims, 1992; Akbarali and Giles, 1993; Espinosa et al., 1998; Hogg et al., 1994; Kleene and Gesteland, 1991; Madrid et al., 2005; Morales et al., 1993) and CaCK (iii) (Greenwood and Large, 1995) in several cell types. Our mathematical model suggests that these channels could participate in the orchestration of the speract-induced $\left[\mathrm{Ca}^{2+}\right]_{\mathrm{i}}$ fluctuations and both are affected by NFA. For the sake of clarity and simplicity, other important changes triggered by speract, such as the $\mathrm{pH}_{\mathrm{i}}$ and cAMP increases are omitted. (B) Alterations of the speract-induced $\left[\mathrm{Ca}^{2+}\right]_{\mathrm{i}}$ fluctuations by NFA (iv) disrupt sperm chemotaxis by exacerbating the $\mathrm{Ca}^{2+}$-dependent turning events that promote the disorientation of the spermatozoa during the straighter swimming episodes (v).

of $V_{\mathrm{m}}$ (Wood et al., 2007). Furthermore, we recently found that the highly specific blocker of CaKC Iberiotoxin at $100 \mathrm{nM}$ increases the period between the $\left[\mathrm{Ca}^{2+}\right]_{\mathrm{i}}$ fluctuations triggered by speract in S. purpuratus spermatozoa (Espinal et al., 2011). If $\mathrm{CaCC}$ and/or CaKC channels participate in the speract signaling pathway, they would open in response to $\mathrm{Ca}^{2+}$ entry, leading to influx of $\mathrm{Cl}^{-}$and/or efflux of $\mathrm{K}^{+}$, and therefore $V_{\mathrm{m}}$ hyperpolarization (Fig. 7A,ii,iii). This hyperpolarization could activate the NCKX that extrudes $\mathrm{Ca}^{2+}$ from the cell, thus shaping the $\left[\mathrm{Ca}^{2+}\right]_{\mathrm{i}}$ increase into a transient fluctuation (Jayantha Gunaratne and Vacquier, 2007; Su and Vacquier, 2002; Nishigaki et al., 2004; Kashikar et al., 2012). This hyperpolarization could also open HCN channels, depolarizing the $V_{\mathrm{m}}$ again, and leading to the opening of $\mathrm{CaV}$ channels and setting up a cycle of $\mathrm{Ca}^{2+}$ influx and efflux through the concerted action of $\mathrm{HCN}, \mathrm{CaV}, \mathrm{CaCC}$ and/or $\mathrm{CaKC}$. The partial block of these channels by NFA may delay the repolarization $\rightarrow$ hyperpolarization phase when the $\left[\mathrm{Ca}^{2+}\right]_{i}$ peaks, and as a consequence delaying the extrusion activity of the NCKX and shaping a $\left[\mathrm{Ca}^{2+}\right]_{\mathrm{i}}$ fluctuation of increased duration and amplitude. Wood et. al, showed that in $S$. purpuratus spermatozoa NFA increases and extends the $V_{\mathrm{m}}$ depolarization following speract addition (Wood et al., 2007). This compound increases the duration, amplitude and interval between successive speract triggered $\left[\mathrm{Ca}^{2+}\right]_{\mathrm{i}}$ fluctuations in S. purpuratus spermatozoa (Wood et al., 2007; Wood et al., 2003) (Fig. 7,iv). As a result, the speract-induced increases in flagellar asymmetry, and the resultant turns of $S$. purpuratus spermatozoa, are more pronounced and prolonged (Wood et al., 2007).

We found that the turning events experienced by NFA-treated L. pictus spermatozoa in a speract gradient are more pronounced due to an increased arc length traveled per turn (Fig. 7B,v). We speculate that the currents flowing through the $\mathrm{CaCC}$ and $\mathrm{CaKC}$ channels are diminished/inhibited due to NFA treatment (Fig. 7,ii, iii); consequently $\mathrm{Ca}^{2+}$ entry through $\mathrm{CaV}$ channels is increased and prolonged (Alvarez et al., 2012), and thus the degree of flagellar bending should be simultaneously increased and prolonged. This latter conjecture is compatible with the extended depolarization following speract addition in the presence of NFA observed in $S$. purpuratus spermatozoa (Wood et al., 2007). We show that the extended arc length traveled per turn due NFA treatment promotes a phase shift of $\pi /$ 2 for the onset of the straighter swimming episodes, as consequence the spermatozoon swims to a less favorable position with respect to the origin of the speract gradient during each straighter swimming episode.

It has been shown that NFA increases the interval between speract-induced $\left[\mathrm{Ca}^{2+}\right]_{\mathrm{i}}$ fluctuations on $S$. purpuratus spermatozoa (Wood et al., 2007; Wood et al., 2003). Even if the $\left[\mathrm{Ca}^{2+}\right]_{\mathrm{i}}$ oscillations remain coupled with the polarity of the chemoattractant gradient, a simple phase shift of the $\left[\mathrm{Ca}^{2+}\right]_{\mathrm{i}}$ oscillator could impair sperm chemotaxis (Fig. 1B). Contrary to our prediction, NFA neither modifies the interval between $\left[\mathrm{Ca}^{2+}\right]_{\mathrm{i}}$ fluctuations induced by the speract gradient in $L$. pictus spermatozoa, nor alters the interval between turning events (supplementary material Fig. S5). Furthermore in the presence of NFA, the $\left[\mathrm{Ca}^{2+}\right]$ i oscillations (Fig. 4) and the associated turning events (supplementary material Fig. S4) were still coupled with the polarity of the chemoattractant concentration gradient suggesting that spermatozoa were unimpaired in their ability to sense and decode this gradient. Such contradictory findings can 
be explained by considering a feedback loop that continuously adjusts the phase of the $\left[\mathrm{Ca}^{2+}\right]_{i}$ oscillator to maintain its coupling with the polarity of the chemoattractant concentration gradient. The increased speract receptor recruitment as sperm swim in the ascending phase of the chemoattractant gradient may feedback to regulate the pacemaker that controls the timing of the $\left[\mathrm{Ca}^{2+}\right]_{\mathrm{i}}$ oscillations (Kashikar et al., 2012). The oscillatory sampling of the chemoattractant concentration gradient, due to their intrinsic periodic swimming behavior, may be enough to correct the expected phase shift of the $\left[\mathrm{Ca}^{2+}\right]_{i}$ oscillations seen in cells stimulated in the absence of a chemoattractant gradient reported by Wood et al. (Wood et al., 2007; Wood et al., 2003). It follows that the impairment of sperm chemotaxis by NFA can be attributed to its modulatory effect on the molecular entities that drive the turning events, and not through alteration of the chemodetection system of concentration gradients. As the speract-induced hyperpolarization first increases $\mathrm{pH}_{\mathrm{i}}$ (Nishigaki et al., 2004) and CatSper, a sperm-specific, pH-dependent $\mathrm{Ca}^{2+}$ channel is found in the S. purpuratus genome (Ren and Xia, 2010), future work must evaluate the role of this channel and its $\mathrm{pH}_{\mathrm{i}}$ regulation in the generation of the $\mathrm{Ca}^{2+}$ bursts, and their sensitivity to NFA.

We conclude that induced alterations to their ionic permeability may disorientate spermatozoa during chemotaxis. It remains to be demonstrated whether this mechanism is relevant during natural chemotactic processes operating during a spermatozoon's journey towards the egg. Combined pharmacology coupled to quantitative and automated methods of analysis of sperm chemotaxis and modeling as the presented here will give more insights regarding the spatiotemporal role of the molecular elements that orchestrate the signal flow that controls sperm swimming behavior.

\section{Materials and Methods}

\section{Materials}

Undiluted L. pictus spermatozoa (Marinus Inc., Long Beach, CA, USA) were obtained by intracoelomic injection of $0.5 \mathrm{M} \mathrm{KCl}$ and stored on ice until used within a day. Artificial seawater (ASW) was 950 to $1000 \mathrm{mOsm}$ and contained (in $\mathrm{mM}): 486 \mathrm{NaCl}, 10 \mathrm{KCl}, 10 \mathrm{CaCl}_{2}, 26 \mathrm{MgCl}_{2}, 30 \mathrm{MgSO}_{4}, 2.5 \mathrm{NaHCO}_{3}, 10$ HEPES and 1 EDTA (pH 7.35). Low $\mathrm{Ca}^{2+}$ ASW was as ASW but $\mathrm{pH} 7.0$ and with $1 \mathrm{mM} \mathrm{CaCl}_{2}$. [Ser5; nitrobenzyl-Gly6]speract, referred to throughout the text as caged speract (CS), was prepared as previously described (Tatsu et al., 2002) Fluo-4-AM and pluronic F-127 were from Molecular Probes, Inc. (Eugene, OR, USA). PolyHEME [poly(2-hydroxyethylmethacrylate)] and NFA \{2-[3(trifluoromethyl)phenyl]aminopyridine-3-carboxylic acid\} were from SigmaAldrich (Toluca, Edo de Mexico, Mexico)

\section{Loading of $\mathrm{Ca}^{2+}$-fluorescent indicator into spermatozoa}

Undiluted spermatozoa were suspended in 10 volumes of low $\mathrm{Ca}^{2+}$ ASW containing $0.2 \%$ pluronic F-127 and $20 \mu \mathrm{M}$ of Fluo-4 AM and incubated for $2.5 \mathrm{~h}$ at $14^{\circ} \mathrm{C}$. Spermatozoa were stored in the dark and on ice until use.

\section{Fluorescence imaging of swimming spermatozoa}

The coverslips were briefly immersed into a $0.1 \% \mathrm{wt} / \mathrm{vol}$ solution of polyHEME in ethanol, hot-air blow-dried to rapidly evaporate the solvent, and mounted on reusable chambers fitting a TC-202 Bipolar temperature controller (Medical Systems Corp.). The temperature plate was mounted on a microscope stage (Eclipse TE-300; Nikon) and maintained at a constant $15^{\circ} \mathrm{C}$. Aliquots of labeled sperm were diluted in ASW and transferred to an imaging chamber (final concentration $\sim 2 \times 10^{5}$ cells $/ \mathrm{ml}$ ). Epifluorescence images were collected with a Nikon Plan Fluor 40× 1.3 NA objective using the Chroma filter set (ex, HQ470/ 40×; DC, 505DCXRU; em, HQ510LP) and recorded on a DV887 iXon EMCCD Andor camera (Andor Bioimaging, NC). Stroboscopic fluorescence illumination was supplied by a Cyan LED no. LXHL-LE5C (Lumileds Lighting LLC, San Jose, USA) synchronized to the exposure output signal of the iXon camera (2 ms illumination per individual exposure). Images were collected with Andor iQ 1.8 software (Andor Bioimaging, NC) at $120 \mathrm{fps}$ in full-chip mode, binning $=4 \times 4$ (observation field of $200 \times 200 \mu \mathrm{m}$ ). The speract gradient was generated via the photolysis of $10 \mathrm{nM}$ caged speract (CS) with a $200 \mathrm{~ms}$ UV pulse delivered through an optical fiber (4 $\mathrm{mm}$ internal diameter) coupled to a Xenon UV lamp (UVICO, Rapp Opto Electronic).

\section{Image processing}

The background fluorescence $\left(F_{0}\right)$ was removed by generating an average pixel intensity time-projection image from the first 360 frames before uncaging which was then subtracted from each frame of the image stack by using the Image calculator tool of ImageJ v1.4 (National Institutes of Health, USA). For Fig. 2, the maximum pixel intensity time projections were created every $3 \mathrm{~s}$ from background-subtracted images before and after the UV flash.

\section{Spermatozoa tracking and detection of $\left[\mathrm{Ca}^{2+}\right]$ i fluctuations}

Spermatozoa were tracked by following the head centroid with the MtrackJ plugin (Meijering, 2006) of ImageJ v 1.4. We scored a $\left[\mathrm{Ca}^{2+}\right]_{\mathrm{i}}$ fluctuation event as the first frame in which the flagellum became visible.

\section{Quantitative and automated analysis of sperm chemotaxis}

We developed a $\mathrm{C} / \mathrm{C}^{2+}$ software called Chemotaxis ${ }_{\mathrm{V} 1 \text { (beta) }}$ to quantitatively analyze the chemotactic responses of marine spermatozoa by calculating the following parameters (supplementary material Fig. S1).

The 'chemotaxis index' (LECI), defined as the negative value of the slope $(L E C I=-M)$ of a least square linear regression $r_{t}=M t+r_{0}$ (Yoshida et al., 2002) where $r_{\mathrm{t}}$ is the distance to the center of the speract gradient, $t$ is the time after UV pulse measured in seconds, with $t \in[0 \mathrm{~s}, 3 \mathrm{~s}$ ] [the reported range where L. pictus spermatozoa experience chemotaxis (Guerrero et al., 2010a)]. Positive LECIs indicate movement towards the chemoattractant source. The center of the speract gradient was estimated by identifying the centroid of the UV flash intensity $x y$ distribution

The relative positioning of the spermatozoa on the chemoattractant gradient at the initiation of each $\left[\mathrm{Ca}^{2+}\right]_{\mathrm{i}}$ fluctuation. For each sperm motility response to speract both the position $\overrightarrow{P(t)}$ and direction $\overrightarrow{D(t)}$ with respect to the chemoattractant gradient center were measured at the beginning of each $\mathrm{Ca}^{2+}$ fluctuation and used to compute $\varphi$ (supplementary material Fig. S6):

$$
\phi(\alpha(t))=\left\{\begin{array}{c}
\frac{\pi}{2}-\alpha(t): \alpha=\left[\frac{\pi}{2}, 0\right) \\
\frac{\pi}{2}+\alpha(t): \alpha=[0, \pi), \\
\frac{S \pi}{2}-\alpha(t): \alpha=\left[\pi, \frac{\pi}{2}\right)
\end{array},\right.
$$

where $\alpha(t)=\arccos \frac{\vec{P} \vec{D}}{|P||D|}$. Values of $\varphi$ between 0 and $\pi$ identify spermatozoa that are swimming away from the center of the chemoattractant gradient, and $\varphi$ values between $\pi$ and $2 \pi$ identify spermatozoa that are swimming toward center of the chemoattractant gradient (supplementary material Fig. S6). High frequency 'noise' due to head wiggling was subtracted from the sperm tracks with a first-order lowpass recursive filter $(f=4 \mathrm{~Hz}$, sampling interval: $s=0.01 \mathrm{~s})$.

The $\varphi$ values were measured at the end of the $200 \mathrm{~ms}$ UV flash ( $\varphi_{\text {UV end }}$ ), at the beginning of the first $\left[\mathrm{Ca}^{2+}\right]_{\mathrm{i}}$ fluctuation $\left(\varphi_{F 1}\right)$, and at the beginning of each $\left[\mathrm{Ca}^{2+}\right]_{\mathrm{i}}$ fluctuation $\left(\varphi_{F}\right)$

The relative positioning of the spermatozoon on the speract gradient at the initiation of each turning event or at the onset of each straighter swimming episode. Sperm motility behavior can be represented as changes in the local path curvature of the swimming trajectory $\kappa$. During the turning events (Turns) the curvature first steeply increases and then decreases below baseline values during the less curved episodes (Runs). Signed path curvatures were computed along time from the $x y$ sperm head coordinates as:

$$
\kappa=\frac{\dot{x} \ddot{y}-\ddot{y} \ddot{x}}{\left(\dot{x}^{2}+\dot{y}^{2}\right)^{3 / 2}},
$$

where $\dot{x}, \dot{y}$ and $\ddot{x}, \ddot{y}$ indicate the first and second order time derivatives. Higher frequency $(>40 \mathrm{~Hz})$ components of $\kappa$ were eliminated.

Given a trajectory $H$ and $\kappa$ its signed curvature, let $\bar{\kappa}$ be the mean path curvature and $c=7$ times the mean SE of $\kappa$ during the motility response to the chemoattractant gradient. We say that $H$ has a turning event in the interval Turn $=[a, b]$, around $t \in$ Turn, if $\kappa(t) \geq \bar{\kappa}+c \forall t \in$ Turn. In the same way, we say that $H$ has a straighter swimming episode in the interval $R u n=\left[a^{\prime}, b^{\prime}\right]$, around $t \in$ Turn, if $\kappa(t) \leq \bar{\kappa}-c \forall t \in R u n$.

The relative positioning of each sperm in the chemoattractant gradient was measured at the onset of the first turning event $\varphi_{T 1}=\varphi(a 1)$, and at the onset of the subsequent turning events $\varphi_{T}=\left(\varphi_{T 2}, \varphi_{T 3}, \ldots, \varphi_{T n}\right)$. In the same way the relative sperm position in the chemoattractant gradient was measured at the onset of the first straighter swimming episode $\varphi_{S 1}=\varphi\left(a^{\prime} 1\right)$, and at the onset of the subsequent straighter swimming episodes $\varphi_{S}=\left(\varphi_{S 2}, \varphi_{S 3}, \ldots, \varphi_{S n}\right)$. 
The mean duration of each turning event $\tau_{T}=b-a$, and each straighter swimming episode $\tau_{S}=b^{\prime}-a^{\prime}$, where $a, a^{\prime}$ and $b, b^{\prime}$ indicate the onset and end times of each turning event or straighter swimming episode, respectively.

The arc length traveled during each turning event. $\mathrm{U}=\varphi(b)-\varphi(a)+2 \pi N^{*}$, where $N^{*}$ is the number of crosses through the nearest point to the center of the cheomattractant gradient during the Turn interval, and $a, b$ indicate the onset and end time of each turning event.

The delay between the end of the $200 \mathrm{~ms}$ UV irradiation period and the onset of the first $\left[\mathrm{Ca}^{2+}\right]_{\mathrm{i}}$ fluctuation $\left(\tau_{F 0}=a_{1}-i\right)$ or to the first turning event $\left(\tau_{T 0}=a_{1}{ }^{\prime}-i\right)$, where $i$ indicates the end time of the UV irradiation period and $a_{1}, a_{1}{ }^{\prime}$ correspond to the onset time of the first $\left[\mathrm{Ca}^{2+}\right]_{\mathrm{i}}$ fluctuation or to the first turning event.

The angular velocity during each turning event $w=v \kappa$, where $v$ is the swimming speed and $\kappa$ is the path curvature.

The source code is available on request from A.G.

\section{Statistical analyses}

Data are presented for a number of individual spermatozoa $(n)$ that were collected from three or more sea urchins. The error bars indicate the standard error of the mean unless specified otherwise. Differences in scalar measurements such as time and distance were assessed using Wilcoxon's non-parametric test. Angles were compared using Rayleigh's test. All statistical tests were performed using R software (R Development Core Team, 2010). The significance level was set at $95 \%$.

\section{Mathematical model}

In a previous work (Espinal et al., 2011), we developed a discrete logical model to describe the temporal dynamics of the speract-activated signaling network in the $S$. purpuratus sea urchin sperm flagellum. Encouraged by the experimental validation of the model predictions (Espinal et al., 2011), here we used this model to investigate in silico the effect on the $\left[\mathrm{Ca}^{2+}\right]_{\mathrm{i}}$ oscillations of altering the NFAsensitive channels present in the sea urchin sperm flagellum.

The model consists of 22 nodes representing the principal components involved, or likely participating, in the signaling cascade: ion channel activities, intracellular ion and molecular concentrations and the membrane potential, amongst others. To analyze the dynamics of the network, we implemented a discrete formulation that is a generalization of the Boolean approach and that has proven to be revealing for the gene regulation dynamics of many systems (Kauffman, 1969; Espinosa-Soto et al., 2004; Albert and Othmer, 2003; Huang and Ingber, 2000; Li et al., 2004), as well as other cell signaling networks (Morris et al., 2010). In this approach, the dynamic state of the network consists of a set of $N$ discrete variables $\left\{\sigma_{1}, \sigma_{2}, \ldots\right.$ $\sigma_{n}$, , each representing the state of a node. For this particular network, most of the variables take on two values, 0 and 1 , depending on whether the corresponding element is absent or present, closed or open, inactive or active, etc. However, an accurate description of the dynamic processes in the network required four nodes to be represented by three-state variables: the membrane potential (hyperpolarized 0 , resting 1 , and depolarized 2); the low and high threshold voltage-gated $\mathrm{Ca}^{2+}$ channels (inactive 0, closed 1, and open 2); and the intracellular calcium concentration $\left[\mathrm{Ca}^{2+}\right]_{\mathrm{i}}$ (basal 0 , tonic 1 and supratonic 2 ). The state of each node $\sigma_{\mathrm{n}}$ is determined by its set of regulators (which are some other nodes that also belong to the network). Let us denote as $\sigma_{\mathrm{n} 1}, \sigma_{\mathrm{n} 2}, \ldots \sigma_{\mathrm{nk}}$ the $k$ regulators of $\sigma_{\mathrm{n}}$. Then, at each time step the value of $\sigma_{\mathrm{n}}$ is given by:

$$
\sigma_{n}(t+1)=F_{n}\left(\sigma_{n 1}(t), \sigma_{n 2}(t), \ldots \sigma_{n k}(t)\right),
$$

where $F_{\mathrm{n}}$ is a regulatory function constructed by taking into account the activating/ inhibiting nature of the regulators. Each node has its own regulatory function. For the construction of these regulatory functions, (which can be found at http://www. fis.unam.mx/research/seaurchin/discrete/), we have made use of all the biological knowledge, mainly of an electrophysiological nature, available to us in the literature and in our own laboratory.

With this model we can observe in silico the effect of blocking certain elements relevant to the pathway. In this paper we consider the case of NFA-sensitive channels: $\mathrm{HCN}, \mathrm{CaKC}$ or CaCC. In order to test the effect of NFA in the network evolution, we deleted all three channels simultaneously. Further studies considering single and/or paired combinations of channel blockade can be found in a companion publication (J.E., A.D., A.G. and G.M.-M., unpublished). Within our model the elimination of node takes place by assigning a zero value to all the regulatory entries in which it participates during the whole network evolution. Hence, each channel will be closed, even if its regulators are activated, thus simulating the inhibitory effect of NFA. We calculated the speract-triggered $\left[\mathrm{Ca}^{2+}\right]_{\mathrm{i}}$ fluctuations averaging them over $10^{5}$ different initial conditions for the whole network (with all nodes present) and compared the result with equivalent calculations performed on the treated networks.

For this type of network all initial conditions lead to a periodic behavior where the network configuration is replicated after a certain number of times. The time required to reach this condition is known as the transient time and the number of iterations between the repeated configurations is the period. These periodic solutions are the attractors of the network dynamics. For the wild-type network
$88.9 \%$ of all speract activated initial conditions lead to a $\left[\mathrm{Ca}^{2+}\right]_{\text {i }}$ period 4 attractor, while the remaining $11.1 \%$ converge to a period 8 attractor. When the three NFA sensitive channels considered in this paper are blocked $92 \%$ of all speract activated initial conditions lead to a $\left[\mathrm{Ca}^{2+}\right]_{\mathrm{i}}$ period 8 attractor different from the previous one, and $8 \%$ go to a new period 9 attractor, thus modifying the temporal characteristics of the $\left[\mathrm{Ca}^{2+}\right]_{\mathrm{i}}$ fluctuations.

\section{Acknowledgements}

The authors thank Dr Tatsu Yoshiro for providing the caged speract and Dr Takuya Nishigaki for his comments and suggestions during the elaboration and analysis of the experiments. G.M. and A.D. thank Consejo Nacional de Ciencia y Tecnología (CONACyT) and DGAPA-UNAM for support during sabbatical leave at the University of Florence, Italy and at the Cell Biology SickKids Hospital, respectively. A.G. and J.E. thank CONACyT for a scholarship.

\section{Author contributions}

A.G., A.D. and C.D.W. conceived the project, participated in the design and drafting of the manuscript. A.G. performed the experiments, created the software for the quantitative, automated analysis of sperm chemotaxis and wrote the paper. J.R. supervised the elaboration of the software. J.E., G.M., A.G., A.D. and C.D.W. created the logical model of the speract signaling pathway. J.E., G.M. and J.C. performed the mathematical model calculations and wrote the corresponding section. J.C. supervised, provided advice and contributed to writing the paper. All authors approved the final manuscript.

\section{Funding}

This work was supported by Consejo Nacional de Ciencia y Tecnología [grant numbers 49113, 128566 to A.D., 132478 to C.D.W.]; Programa de Apoyo a Proyectos de Investigación e Innovación Technológica [grant numbers IN211809, DGAPA/IXTLI IX200910 to A.D., IN223810 to C.D.W., IN109210-F to G.M.]; and Fundação para a Ciência e a Tecnologia [grant number PTDC/SALOBD/69928/2006 to J.C.].

\section{Supplementary material available online at} http://jcs.biologists.org/lookup/suppl/doi:10.1242/jcs.121442/-/DC1

\section{References}

Akbarali, H. I. and Giles, W. R. (1993). Ca2+ and $\mathrm{Ca}(2+)$-activated Cl- currents in rabbit oesophageal smooth muscle. J. Physiol. 460, 117-133.

Albert, R. and Othmer, H. G. (2003). The topology of the regulatory interactions predicts the expression pattern of the segment polarity genes in Drosophila melanogaster. J. Theor. Biol. 223, 1-18.

Alvarez, L., Dai, L., Friedrich, B. M., Kashikar, N. D., Gregor, I., Pascal, R. and Kaupp, U. B. (2012). The rate of change in $\mathrm{Ca}(2+)$ concentration controls sperm chemotaxis. J. Cell Biol. 196, 653-663.

Böhmer, M., Van, Q., Weyand, I., Hagen, V., Beyermann, M., Matsumoto, M., Hoshi, M., Hildebrand, E. and Kaupp, U. B. (2005). Ca2+ spikes in the flagellum control chemotactic behavior of sperm. EMBO J. 24, 2741-2752.

Bönigk, W., Loogen, A., Seifert, R., Kashikar, N., Klemm, C., Krause, E., Hagen, V., Kremmer, E., Strünker, T. and Kaupp, U. B. (2009). An atypical CNG channel activated by a single cGMP molecule controls sperm chemotaxis. Sci. Signal. 2, ra68.

Cheng, L. and Sanguinetti, M. C. (2009). Niflumic acid alters gating of HCN2 pacemaker channels by interaction with the outer region of S4 voltage sensing domains. Mol. Pharmacol. 75, 1210-1221.

Cook, S. P., Brokaw, C. J., Muller, C. H. and Babcock, D. F. (1994). Sperm chemotaxis: egg peptides control cytosolic calcium to regulate flagellar responses. Dev. Biol. 165, 10-19.

Darszon, A., Guerrero, A., Galindo, B. E., Nishigaki, T. and Wood, C. D. (2008). Sperm-activating peptides in the regulation of ion fluxes, signal transduction and motility. Int. J. Dev. Biol. 52, 595-606.

Darszon, A., Nishigaki, T., Beltran, C. and Treviño, C. L. (2011). Calcium channels in the development, maturation, and function of spermatozoa. Physiol. Rev. 91, 13051355

Espinal, J., Aldana, M., Guerrero, A., Wood, C., Darszon, A. and Martínez-Mekler, G. (2011). Discrete dynamics model for the speract-activated Ca2+ signaling network relevant to sperm motility. PLOS ONE 6, e22619.

Espinosa, F., de la Vega-Beltrán, J. L., López-González, I., Delgado, R., Labarca, P. and Darszon, A. (1998). Mouse sperm patch-clamp recordings reveal single 
Cl- channels sensitive to niflumic acid, a blocker of the sperm acrosome reaction. FEBS Lett. 426, 47-51.

Espinosa-Soto, C., Padilla-Longoria, P. and Alvarez-Buylla, E. R. (2004). A gene regulatory network model for cell-fate determination during Arabidopsis thaliana flower development that is robust and recovers experimental gene expression profiles Plant Cell 16, 2923-2939.

Friedrich, B. M. and Jülicher, F. (2007). Chemotaxis of sperm cells. Proc. Natl. Acad. Sci. USA 104, 13256-13261.

Galindo, B. E., Neill, A. T. and Vacquier, V. D. (2005). A new hyperpolarizationactivated, cyclic nucleotide-gated channel from sea urchin sperm flagella. Biochem. Biophys. Res. Commun. 334, 96-101.

Galindo, B. E., de la Vega-Beltrán, J. L., Labarca, P., Vacquier, V. D. and Darszon, A. (2007). Sp-tetraKCNG: A novel cyclic nucleotide gated $\mathrm{K}(+)$ channel. Biochem. Biophys. Res. Commun. 354, 668-675.

Gauss, R., Seifert, R. and Kaupp, U. B. (1998). Molecular identification of a hyperpolarization-activated channel in sea urchin sperm. Nature 393, 583-587.

González-Martínez, M. and Darszon, A. (1987). A fast transient hyperpolarization occurs during the sea urchin sperm acrosome reaction induced by egg jelly. FEBS Lett. 218, 247-250.

Granados-Gonzalez, G., Mendoza-Lujambio, I., Rodriguez, E., Galindo, B. E., Beltrán, C. and Darszon, A. (2005). Identification of voltage-dependent $\mathrm{Ca} 2+$ channels in sea urchin sperm. FEBS Lett. 579, 6667-6672.

Greenwood, I. A. and Large, W. A. (1995). Comparison of the effects of fenamates on Ca-activated chloride and potassium currents in rabbit portal vein smooth muscle cells. Br. J. Pharmacol. 116, 2939-2948.

Guerrero, A., Nishigaki, T., Carneiro, J., Tatsu, Y., Wood, C. D. and Darszon, A. (2010a). Tuning sperm chemotaxis by calcium burst timing. Dev. Biol. 344, 52-65.

Guerrero, A., Wood, C. D., Nishigaki, T., Carneiro, J. and Darszon, A. (2010b) Tuning sperm chemotaxis. Biochem. Soc. Trans. 38, 1270-1274.

Hansbrough, J. R. and Garbers, D. L. (1981). Speract. Purification and characterization of a peptide associated with eggs that activates spermatozoa. J. Biol. Chem. 256, 1447-1452.

Hogg, R. C., Wang, Q. and Large, W. A. (1994). Action of niflumic acid on evoked and spontaneous calcium-activated chloride and potassium currents in smooth muscle cells from rabbit portal vein. Br. J. Pharmacol. 112, 977-984.

Huang, S. and Ingber, D. E. (2000). Shape-dependent control of cell growth, differentiation, and apoptosis: switching between attractors in cell regulatory networks. Exp. Cell Res. 261, 91-103.

Janssen, L. J. and Sims, S. M. (1992). Acetylcholine activates non-selective cation and chloride conductances in canine and guinea-pig tracheal myocytes. J. Physiol. 453, 197-218.

Jayantha Gunaratne, H. and Vacquier, V. D. (2007). Sequence, annotation and developmental expression of the sea urchin $\mathrm{Ca}(2+)$-ATPase family. Gene 397, 67-75.

Kashikar, N. D., Alvarez, L., Seifert, R., Gregor, I., Jäckle, O., Beyermann, M., Krause, E. and Kaupp, U. B. (2012). Temporal sampling, resetting, and adaptation orchestrate gradient sensing in sperm. J. Cell Biol. 198, 1075-1091.

Kauffman, S. A. (1969). Metabolic stability and epigenesis in randomly constructed genetic nets. J. Theor. Biol. 22, 437-467.

Kaupp, U. B., Solzin, J., Hildebrand, E., Brown, J. E., Helbig, A., Hagen, V., Beyermann, M., Pampaloni, F. and Weyand, I. (2003). The signal flow and motor response controling chemotaxis of sea urchin sperm. Nat. Cell Biol. 5, 109-117.

Kaupp, U. B., Kashikar, N. D. and Weyand, I. (2008). Mechanisms of sperm chemotaxis. Annu. Rev. Physiol. 70, 93-117.

Kleene, S. J. and Gesteland, R. C. (1991). Calcium-activated chloride conductance in frog olfactory cilia. J. Neurosci. 11, 3624-3629.

Li, F., Long, T., Lu, Y., Ouyang, Q. and Tang, C. (2004). The yeast cell-cycle network is robustly designed. Proc. Natl. Acad. Sci. USA 101, 4781-4786.

Li, L., Ma, K. T., Zhao, L. and Si, J. Q. (2008). Niflumic acid hyperpolarizes the smooth muscle cells by opening BK(Ca) channels through ryanodine-sensitive $\mathrm{Ca}(2+)$ release in spiral modiolar artery. Sheng Li Xue Bao 60, 743-750.

Madrid, R., Delgado, R. and Bacigalupo, J. (2005). Cyclic AMP cascade mediates the inhibitory odor response of isolated toad olfactory receptor neurons. J. Neurophysiol. 94, 1781-1788.

Meijering, E. (2006). MTrackJ: A Java program for manual object tracking.

Miller, R. (1985). Sperm Chemo-orientation in the Metazoa, Vol. 2 of Biology of fertilization, pp. 275-337. New York, NY: Academic Press.
Miller, R. and Brokaw, C. (1970). Chemotactic turning behaviour of Tubularia spermatozoa. J. Exp. Biol. 52, 699-706.

Morales, E., de la Torre, L., Moy, G. W., Vacquier, V. D. and Darszon, A. (1993) Anion channels in the sea urchin sperm plasma membrane. Mol. Reprod. Dev. 36, 174-182.

Morris, M. K., Saez-Rodriguez, J., Sorger, P. K. and Lauffenburger, D. A. (2010). Logic-based models for the analysis of cell signaling networks. Biochemistry 49, 3216-3224.

Nishigaki, T., Zamudio, F. Z., Possani, L. D. and Darszon, A. (2001). Time-resolved sperm responses to an egg peptide measured by stopped-flow fluorometry. Biochem. Biophys. Res. Commun. 284, 531-535.

Nishigaki, T., Wood, C. D., Tatsu, Y., Yumoto, N., Furuta, T., Elias, D., Shiba, K., Baba, S. A. and Darszon, A. (2004). A sea urchin egg jelly peptide induces a cGMP-mediated decrease in sperm intracellular $\mathrm{Ca}(2+)$ before its increase. Dev. Biol. 272, 376-388

Pacaud, P., Loirand, G., Lavie, J. L., Mironneau, C. and Mironneau, J. (1989) Calcium-activated chloride current in rat vascular smooth muscle cells in short-term primary culture. Pflugers Arch. 413, 629-636.

R Development Core Team (2010). R: A Language and Environment for Statistical Computing. R Foundation for Statistical Computing, Vienna, Austria. ISBN 3900051-07-0.

Ren, D. and Xia, J. (2010). Calcium signaling through CatSper channels in mammalian fertilization. Physiology (Bethesda) 25, 165-175.

Satoh, T. O. and Yamada, M. (2001). Niflumic acid reduces the hyperpolarizationactivated current (I(h)) in rod photoreceptor cells. Neurosci. Res. 40, 375-381.

Schackmann, R. W., Christen, R. and Shapiro, B. M. (1981). Membrane potential depolarization and increased intracellular $\mathrm{pH}$ accompany the acrosome reaction of sea urchin sperm. Proc. Natl. Acad. Sci. USA 78, 6066-6070.

Shiba, K., Baba, S. A., Inoue, T. and Yoshida, M. (2008). Ca2+ bursts occur around a local minimal concentration of attractant and trigger sperm chemotactic response. Proc. Natl. Acad. Sci. USA 105, 19312-19317.

Strünker, T., Weyand, I., Bönigk, W., Van, Q., Loogen, A., Brown, J. E., Kashikar, N., Hagen, V., Krause, E. and Kaupp, U. B. (2006). A K+-selective cGMP-gated ion channel controls chemosensation of sperm. Nat. Cell Biol. 8, 1149-1154.

Su, Y. H. and Vacquier, V. D. (2002). A flagellar $\mathrm{K}(+)$-dependent $\mathrm{Na}(+) / \mathrm{Ca}(2+)$ exchanger keeps $\mathrm{Ca}(2+)$ low in sea urchin spermatozoa. Proc. Natl. Acad. Sci. USA 99, 6743-6748.

Suzuki, N. (1995). Structure, function and biosynthesis of sperm-activating peptides and fucose sulfate glycoconjugate in the extracellular coat of sea urchin eggs. Zoolog. Sci. 12, 13-27.

Suzuki, N. and Garbers, D. L. (1984). Stimulation of sperm respiration rates by speract and resact at alkaline extracellular pH. Biol. Reprod. 30, 1167-1174.

Suzuki, N., Nomura, K., Ohtake, H. and Isaka, S. (1981). Purification and the primary structure of sperm-activity peptides from the jelly coat of sea urchin eggs. Biochem. Biophys. Res. Commun. 99, 1238-1244.

Tatsu, Y., Nishigaki, T., Darszon, A. and Yumoto, N. (2002). A caged spermactivating peptide that has a photocleavable protecting group on the backbone amide. FEBS Lett. 525, 20-24

Ward, G. E., Brokaw, C. J., Garbers, D. L. and Vacquier, V. D. (1985). Chemotaxis of Arbacia punctulata spermatozoa to resact, a peptide from the egg jelly layer. J. Cell Biol. 101, 2324-2329.

White, M. M. and Aylwin, M. (1990). Niflumic and flufenamic acids are potent reversible blockers of $\mathrm{Ca} 2(+)$-activated $\mathrm{Cl}-$ channels in Xenopus oocytes. Mol. Pharmacol. 37, 720-724.

Wood, C. D., Darszon, A. and Whitaker, M. (2003). Speract induces calcium oscillations in the sperm tail. J. Cell Biol. 161, 89-101.

Wood, C. D., Nishigaki, T., Furuta, T., Baba, S. A. and Darszon, A. (2005). Real-time analysis of the role of $\mathrm{Ca}(2+)$ in flagellar movement and motility in single sea urchin sperm. J. Cell Biol. 169, 725-731.

Wood, C. D., Nishigaki, T., Tatsu, Y., Yumoto, N., Baba, S. A., Whitaker, M. and Darszon, A. (2007). Altering the speract-induced ion permeability changes that generate flagellar $\mathrm{Ca} 2+$ spikes regulates their kinetics and sea urchin sperm motility. Dev. Biol. 306, 525-537.

Yoshida, M., Murata, M., Inaba, K. and Morisawa, M. (2002). A chemoattractant for ascidian spermatozoa is a sulfated steroid. Proc. Natl. Acad. Sci. USA 99, 1483114836 . 